\title{
Vasoplegia after pediatric cardiac transplantation in patients supported with a continuous flow ventricular assist device
}

\author{
Loren D. Sacks, MD, ${ }^{\mathrm{a}}$ Seth A. Hollander, MD, ${ }^{\mathrm{a}}$ Yulin Zhang, PhD, ${ }^{\mathrm{b}}$ Kathleen R. Ryan, MD, \\ Mackenzie A. Ford, MD, ${ }^{a}$ Katsuhide Maeda, MD, ${ }^{b}$ Jenna M. Murray, NP, \\ Christopher S. Almond, MD, MPH, and David M. Kwiatkowski, MD ${ }^{\mathrm{a}}$
}

\section{ABSTRACT}

Objective: To determine the association between continuous flow ventricular assist devices and the incidence of vasoplegia following orthotopic heart transplant in children. Moreover, to propose a novel clinical definition of vasoplegia for use in pediatric populations.

Methods: This is a single-center, retrospective cohort study set in the cardiovascular intensive care unit of a tertiary children's hospital. All patients aged 3 years and older who underwent orthotopic heart transplant at Stanford University between April 1, 2014, and July 31, 2017, were included. Vasoplegia was defined by the use of vasoconstrictive medication, diastolic hypotension, preserved systolic heart function, and absence of infection or right atrial pressure or central venous pressure $<5 \mathrm{~mm} \mathrm{Hg}$.

Results: Of 44 eligible patients, 21 were supported using a continuous flow ventricular assist device. Following heart transplant, 14 patients (32\%) developed vasoplegia by the study definition. Development of vasoplegia was associated with pretransplant use of a continuous flow ventricular assist device $(52 \%$ vs $13 \%)$ with a relative risk of 4.02 (95\% confidence interval, 1.30-12.45; $P=.009)$. No other variables were predictive of vasoplegia in univariable analysis. Presence of vasoplegia was not associated with adverse outcomes, although there were trends towards higher incidence of acute kidney injury and increased length of hospital stays.

Conclusions: Children receiving continuous flow ventricular assist device support are at increased risk for vasoplegia following orthotopic heart transplant, using a novel definition of vasoplegia. Anticipation of this complication will allow for prompt intervention, thereby minimizing hemodynamic instability and impact on patient outcomes. (J Thorac Cardiovasc Surg 2019;157:2433-40)

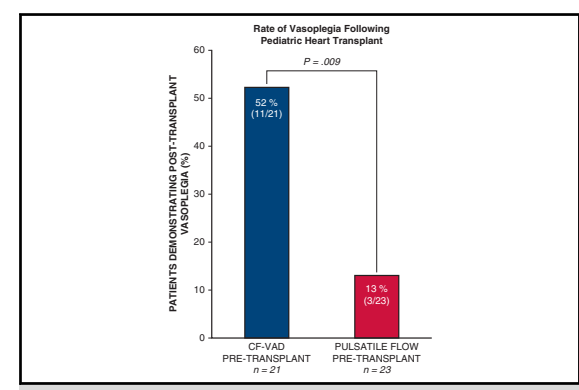

Vasoplegia rates after heart transplant in children supported with continuous flow VADs.

\section{Central Message}

Children supported with continuous flow ventricular assist devices are at significantly increased risk for vasoplegia following heart transplant using a novel, and reproducible, clinical definition.

\section{Perspective}

Although continuous flow VAD use is associated with vasoplegia after heart transplant in adults, this has not been studied in pediatric patients. Moreover, vasoplegia is poorly defined in pediatric populations. This study provides the first reproducible, clinical definition of pediatric vasoplegia and demonstrates a strong link between pre-transplant continuous flow VAD use and vasoplegia after heart transplant

See Commentaries on pages 2441 and 2443.

\footnotetext{
From the Departments of ${ }^{\mathrm{a} P e d i a t r i c s-C a r d i o l o g y}$ and ${ }^{\mathrm{b}}$ Cardiothoracic Surgery-Pediatric Cardiac Surgery, Stanford University, Palo Alto, Calif; and ${ }^{\mathrm{c}}$ Pediatric Advanced Cardiac Therapies-Mechanical Circulatory Support, Lucile Packard Children's Hospital Stanford, Palo Alto, Calif.

Received for publication April 17, 2018; revisions received Jan 22, 2019; accepted for publication Jan 27, 2019; available ahead of print March 29, 2019.

Address for reprints: Loren D. Sacks, MD, Department of Pediatrics-Cardiology, Stanford University, 750 Welch Rd, Ste 321, Palo Alto, CA 94304 (E-mail: 1sacks@stanford.edu).

0022-5223/\$36.00

Copyright $(\underset{)}{ } 2019$ by The American Association for Thoracic Surgery

https://doi.org/10.1016/j.jtcvs.2019.01.100
}

Heart transplantation is used increasingly for the treatment of end-stage heart disease in children, with expected increases in the pre- and posttransplant risk profiles of patients managed in high-volume centers. In the current era,

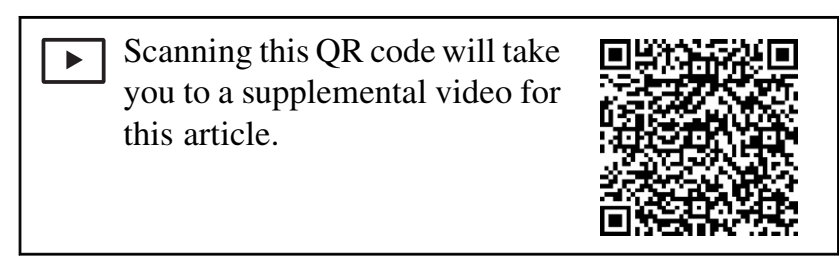



Abbreviations and Acronyms
$\mathrm{AKI}=$ acute kidney injury
$\mathrm{CF}=$ continuous flow
$\mathrm{ICU}=$ intensive care unit
$\mathrm{OHT}=$ orthotopic heart transplant
$\mathrm{VAD}=$ ventricular assist device

The aim of this study was to provide a standardized definition of vasoplegia and to utilize that definition to establish risk factors for vasoplegia following orthotopic heart transplant (OHT) in children. The primary objective was to determine whether the use of a CF-VAD as a bridge to transplant is associated with a greater incidence of vasoplegia after OHT, to establish risk factors for post-OHT vasoplegia, and to examine the association between vasoplegia and post-OHT adverse outcomes.

\section{METHODS}

\section{Study Design and Population}

This was a single-center retrospective study of all consecutive patients between ages 3 and 18 years who underwent OHT at Lucile Packard Children's Hospital Stanford from April 1, 2014, to July 31, 2017. Because the study was designed to evaluate vasoplegia specifically following heart transplant, patients were excluded if they underwent multiple organ transplants. In addition, those who required postoperative extracorporeal membrane oxygenation within the first 24 hours following heart transplantation were excluded because the use of this technology negated the use of clinical factors inherent to the study's definition of vasoplegia. Patients younger than age 3 years were excluded because the standard management of the institution would avoid the use of a CF-VAD, the primary variable of evaluation. Had patients younger than age 3 years been included, 15 subjects would have been added to the pulsatile cohort, compared with 1 in the CF-VAD cohort (Figure 1), biasing the pulsatile cohort toward younger patients. The age cutoff was instituted to eliminate this bias and to assess cohorts of similar ages. All remaining patients, including repeat transplantations, were included. However, the use of CF-VAD support is not without some risk. Specifically, in adults, several studies have noted an increased incidence of vasoplegia following heart transplantation after CF-VAD support. ${ }^{6-8}$ Vasoplegia is known to carry a poor prognosis in adults, with prolonged intensive care unit (ICU) stays, prolonged need for mechanical ventilation, and in some reports, mortality rates as high as $25 \% .{ }^{9,10}$ This phenomenon of hypotension with low systemic vascular resistance can be especially challenging to manage following transplantation because the newly transplanted right ventricle is susceptible to injury with the aggressive fluid resuscitation often required for management of this state.

Although various criteria exist in the adult literature, definitions of vasoplegia typically include not only systemic hypotension, but also a normal/elevated cardiac index and depressed systemic vascular resistance as measured by pulmonary artery catheter and thermodilution. ${ }^{10,11}$ In part due to smaller vessel size, the use of pulmonary artery catheters in pediatric intensive care is rare. ${ }^{11,12}$ The definition of vasoplegia in children is therefore subjective and lacks consistency. Most ICU providers agree that a combination of hypotension and the need for a vasoconstricting agent defines a vasoplegic state, regardless of the etiology. However, to effectively study the effects of various conditions on the development and management of primary vasoplegia in children, unifying criteria must be established.

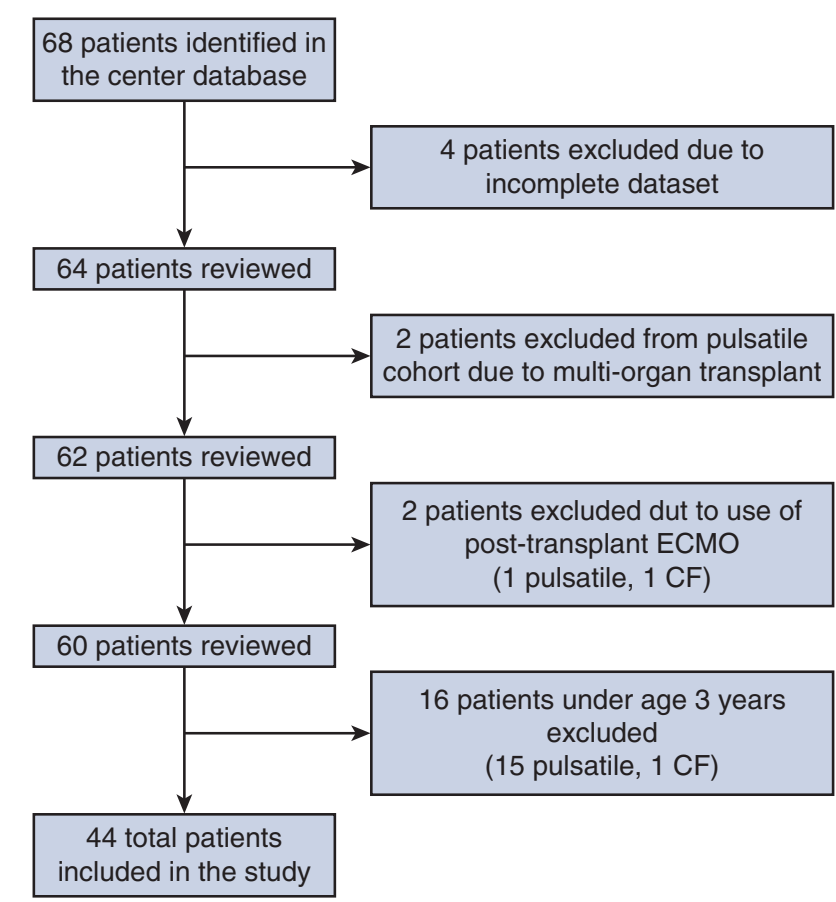

FIGURE 1. A total of 68 patients were identified in the institutional database who had undergone heart transplantation during the era studied. After exclusion of subjects as noted, a total of 44 patients were included in final data analysis. ECMO, Extracorporeal membrane oxygenation; $C F$, continuous flow. 


\section{Data Collection}

Preoperative data included demographic data, primary cardiac disease, use of inotropic infusions (milrinone, dopamine, or epinephrine), use of VADs, use of medications such as angiotensin-converting enzyme inhibitors and/or amiodarone, and serum creatinine level immediately before transplantation. Intraoperative data included donor heart ischemic time and use of plasmapheresis. Postoperative data included use of inhaled nitric oxide, inotropic and vasoactive infusions, frequency of blood transfusion, serum creatinine level, hemodynamic data for 72 hours, echocardiographic data on postoperative day 1 and postoperative day 2, blood and urine cultures obtained for 5 days, ventilator use, and hospital stays. All data collection and analysis were performed under acceptance of our institutional review board. Data were collected and stored in RedCAP version 6.9.7 (University of Alberta, Edmonton, Alberta, Canada), a Web-based application designed to support data capture for research studies. ${ }^{13}$

\section{Definitions}

Patients were considered to have primary vasoplegia through the satisfaction of all 4 of the following criteria: use of vasopressor medication (vasopressin or norepinephrine) or high-dose inotrope (epinephrine $\geq 0.08 \mu \mathrm{g} / \mathrm{kg} / \mathrm{min}$ or dopamine $\geq 8 \mu \mathrm{g} / \mathrm{kg} / \mathrm{min}$ ); diastolic blood pressure measured from an indwelling arterial line below the 10th percentile for age and height percentile ${ }^{14,15}$ within the same day as vasopressor initiation or escalation; systolic ejection fraction $>60 \%$ in the first 72 hours after transplant and no evidence of positive blood or urine cultures within the first 5 postoperative days; and right atrial pressure $\geq 5 \mathrm{~mm} \mathrm{Hg}$ (Figure 2). Diastolic blood pressure was focused on because vasoplegia primarily affects this variable.

Patients were considered to have acute kidney injury (AKI) if they met classification for stage 2 to 3 AKI by Kidney Disease Improving Global Outcomes serum creatinine criteria, which requires at least a doubling of serum creatinine level. ${ }^{16}$

\section{Statistical Analysis}

Because samples were not normally distributed, continuous variables were summarized as median with interquartile range (IQR). Categorical variables were summarized as frequency and percent. Clinical characteristics and outcome data were compared across groups using Fisher exact test or Wilcoxon rank sum test as appropriate. Bootstrap method was used to evaluate potential risk factors for vasoplegia. The following variables were evaluated: gender, age, height, weight, single ventricle physiology, congenital heart disease, angiotensin-converting enzyme inhibitors,

Use of any one of the following:

-Vasopressin

-Norepinephrine

-High-dose Epinephrine ( $\geq 0.08 \mu \mathrm{g} / \mathrm{kg} / \mathrm{min})$

-High-dose Dopamine ( $\geq 8 \mu \mathrm{g} / \mathrm{kg} / \mathrm{min})$

-AND-

Diastolic blood pressure below $10^{\text {th }}$ percentile

-WITHOUT-

Systolic Ejection Fraction $<60 \%$ on Echocardiogram Positive Blood or Urine Culture

Right Atrial Pressure or CVP $<5 \mathrm{~mm} \mathrm{Hg}$

FIGURE 2. Pediatric primary vasoplegia is defined by the criteria displayed. Subjects must meet all criteria to be categorized as experiencing vasoplegia after heart transplant. $C V P$, Central venous pressure. spironolactone, amiodarone, inotropy, creatinine, hemoglobin, cardiopulmonary bypass, plasmaphresis, graft ischemic time, and CF-VAD. Ten thousand bootstrap samples of equal size were generated by resampling with replacement. For each bootstrap sample, univariable logistic regression models were fitted with vasoplegia as the dependent variable and each of the variables listed above as the independent variable. A variable would be selected as a potential risk factor if it was significant at the $P=.05$ level using the likelihood ratio test. The frequencies of each variable being selected out of the 10,000 bootstrap samples were calculated and a variable would be considered as a risk factor for vasoplegia if its frequency was $>50 \%$. Variables were also evaluated using bivariable logistic regression models with $\mathrm{CF}-\mathrm{VAD}$ as 1 of the 2 independent variables in bootstrap samples with CF-VAD selected. All statistical analysis was performed using IBM SPSS Statistics version 25 software (IBM-SPSS Inc, Armonk, NY) and R version 3.5.0 (R Foundation for Statistical Computing, Vienna, Austria).

\section{RESULTS}

\section{Baseline Characteristics}

A total of 68 patients underwent OHT during the study period. Of these, 44 patients $(65 \%)$ met criteria for study inclusion. Of 44 patients included, 21 (48\%) were bridged to transplant using a CF-VAD (HeartWare Ventricular Assist System; HeartWare Inc, Framingham, Mass), 1 of which had biventricular support. Of 23 patients $(52 \%)$ receiving pulsatile support, 2 were bridged to transplant using a pulsatile flow device (Excor $25 \mathrm{~mL}$; Berlin Heart, Berlin, Germany), including 1 with biventricular support and the remaining 21 received medical support for their native hearts until the time of transplantation. Sixteen patients with baseline single-ventricle physiology were included, 4 in the CF-VAD cohort and 12 in the pulsatile support cohort. Of all single-ventricle patients included, 3 had proteinlosing enteropathy, and 4 had plastic bronchitis, whereas 1 patient had both. All 6 of these patients had pulsatile blood flow support pretransplant. Patients with CF-VAD support had greater height and weight and demonstrated lower pretransplant hemoglobin levels than patients in the pulsatile cohort. The pulsatile and continuous flow groups were similar in their preoperative creatinine, use of plasmapheresis, posttransplant inhaled nitric oxide use, and preoperative inotropic medications. Posttransplant inotropic requirements, as measured by the vasoactive-inotropic score $^{17,18}$ were similar in the pulsatile and CF cohorts because were the lowest daily right atrial pressure. The vasoactive-inotropic score of patients meeting criteria for vasoplegia was significantly higher than that of nonvasoplegic patients at 6 hours posttransplant ( 16.5 vs $13 ; P=.003$ ). Demographic and baseline characteristics of patients according to preoperative pulsatile and $\mathrm{CF}$ circulations are shown in Table 1.

\section{Risk Factors for Vasoplegia}

Fourteen $(32 \%)$ patients developed postoperative vasoplegia, 11 of whom $(79 \%)$ were supported with a

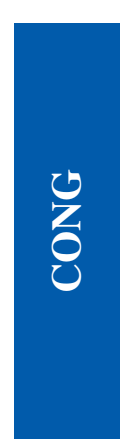


TABLE 1. Characteristics of the study population

\begin{tabular}{|c|c|c|c|c|}
\hline Characteristic & Total $(n=44)$ & Pulsatile flow $(n=23)$ & Continuous flow $(n=21)$ & $P$ value \\
\hline Female sex & $15(34)$ & $8(35)$ & $7(33)$ & $>.999$ \\
\hline Age (y) & $13.6(9.5-15.7)$ & $12.6(5.2-15.8)$ & $13.6(11.7-15.5)$ & .307 \\
\hline Height $(\mathrm{cm})$ & $149(126-166)$ & $138(101-158)$ & $158(142-168)$ & .031 \\
\hline Weight (kg) & $43.7(29.5-68.8)$ & $36.1(16.1-55.4)$ & $56.5(43.6-72.6)$ & .008 \\
\hline Single ventricle & $16(36)$ & $12(52)$ & $4(19)$ & .031 \\
\hline $\begin{array}{l}\text { Primary diagnosis } \\
\text { CHD } \\
\text { CM } \\
\text { Re-transplant }\end{array}$ & $\begin{array}{c}19(43) \\
23(52) \\
2(5)\end{array}$ & $\begin{array}{c}12(52) \\
9(39) \\
2(9)\end{array}$ & $\begin{array}{c}7(33) \\
14(67) \\
0(0)\end{array}$ & .119 \\
\hline \multicolumn{5}{|l|}{ Medication } \\
\hline ACE inhibitors & $26(59)$ & $10(43)$ & $16(76)$ & .036 \\
\hline Spironolactone & $31(70)$ & $18(78)$ & $13(62)$ & .325 \\
\hline Amiodarone & $9(20)$ & $1(4)$ & $8(38)$ & .008 \\
\hline Inotropy & $10(23)$ & $7(30)$ & $3(14)$ & .287 \\
\hline Creatinine (mg/dL) & $0.69(0.51-0.80)$ & $0.58(0.41-0.76)$ & $0.70(0.64-0.80)$ & .064 \\
\hline Hemoglobin (g/dL) & $11.9(11.2-13.6)$ & $13.4(12.3-14.4)$ & $11.1(10.2-11.8)$ & $<.001$ \\
\hline CPB time (min) & $188(159-208)$ & $195(170-220)$ & $172(154-195)$ & .084 \\
\hline Plasmaphresis & $27(61)$ & $14(61)$ & $13(62)$ & $>.999$ \\
\hline Graft ischemic time (min) & $222(200-238)$ & $222(206-238)$ & $227(192-238)$ & .953 \\
\hline iNO posttransplant & $23(52)$ & $9(39)$ & $14(67)$ & .080 \\
\hline EF posttransplant $(\%)$ & $67(65-71)$ & $68(66-72)$ & $67(64-70)$ & .526 \\
\hline VIS at $6 \mathrm{~h}$ & $13.0(11.5-15.1)$ & $13.0(11.8-15.0)$ & $14.0(11.0-16.0)$ & .786 \\
\hline VIS at $12 \mathrm{~h}$ & $13.0(11.4-15.0)$ & $13.0(11.2-15.0)$ & $13.0(11.5-15.5)$ & .741 \\
\hline VIS at $24 \mathrm{~h}$ & $11.2(10.5-14.0)$ & $12.0(11.0-14.2)$ & $11.0(10.0-13.0)$ & .103 \\
\hline RA pressure on day $1(\mathrm{~mm} \mathrm{Hg})$ & $6(5-9)$ & $6(5-8)$ & $7(5-10)$ & .414 \\
\hline RA pressure on day $2(\mathrm{~mm} \mathrm{Hg})$ & $10(7-12)$ & $10(8-13)$ & $8(6-10)$ & .137 \\
\hline RA pressure on day $3(\mathrm{~mm} \mathrm{Hg})$ & $8(7-11)$ & $9(8-11)$ & $8(7-11)$ & .395 \\
\hline ATG within $3 \mathrm{~d}$ & $42(95)$ & $22(96)$ & $20(95)$ & $>.999$ \\
\hline
\end{tabular}

Values are presented as $\mathrm{n}(\%)$ or median (interquartile range). $C H D$, Congenital heart disease; $C M$, cardiomyopathy; $A C E$, angiotensin converting enzyme; $C P B$, cardiopulmonary bypass; $i N O$, inhaled nitric oxide; $E F$, ejection fraction; $V I S$, vasoactive-inotropic score; $R A$, right atrial; $A T G$, antithymocyte globulin.

CF-VAD. Occurrence of vasoplegia was associated with pretransplant use of a CF-VAD (52\% vs $13 \%)$ with a relative risk of $4.02(95 \%$ confidence interval, $1.30-12.45$; $P=.009$ ) (Figure 3). Among 10,000 bootstrap samples of size 44 this univariable logistic regression model had CFVAD being significant at the $5 \%$ level $83 \%$ of the time, whereas all other variables others were significant only $7 \%$ to $16 \%$ (Table 2 ) of the time, identifying CF-VAD as the most influential risk factor for vasoplegia. Given the low number $(\mathrm{n}=2)$ of pulsatile VAD patients included in this study population, these analyses were repeated after the exclusion of those subjects. Vasoplegia remained highly associated with the use of a CF-VAD ( $52 \%$ vs $14 \%)$ with a relative risk of 3.67 (95\% confidence interval, 1.19-11.29; $P=.02$ ). Univariable logistic regression models continued to demonstrate CF-VAD use as significantly associated with vasoplegia $79 \%$ of the time, with no other variable reaching significance more than $13 \%$ of the time.

To examine whether a variable would show significant association with vasoplegia in the presence of CF-VAD, a bivariable models were fitted using CF-VAD as 1 of the 2 independent variables in bootstrap samples with CF-VAD being significant. Single-ventricle physiology and pretransplant hemoglobin level were significant $54 \%$ and $51 \%$ of the time, respectively. All other variables were significant only $6 \%$ to $28 \%$ of the time. Further analysis showed that the association between pretransplant hemoglobin and vasoplegia was mainly due to the higher hemoglobin levels in single-ventricle patients. In the subsets of singleventricle patients and biventricular patients, the association between hemoglobin and vasoplegia was no longer statistically significant. 


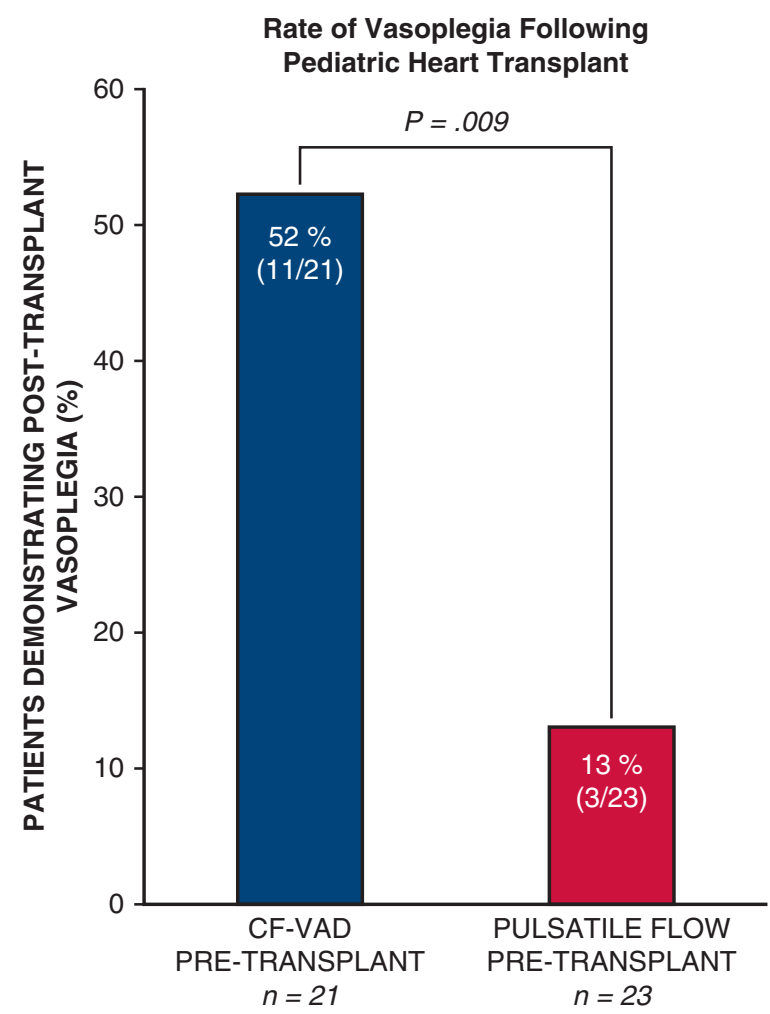

FIGURE 3. Following heart transplant, 11 out of 21 patients (52\%) supported pretransplant with a continuous flow ventricular assist device ( $C F-V A D)$ developed vasoplegia. This is compared with 3 out of 23 patients $(13 \%)$ who were supported with pulsatile flow pretransplant. The difference is significant $(P=.009)$.

\section{Description of Patients With Vasoplegia}

Of 14 patients meeting criteria for a diagnosis of vasoplegia, 11 patients $(78 \%)$ satisfied criteria within the first 12 hours following transplant. Eight of those 14 patients $(64 \%)$ were prescribed high-dose epinephrine, receiving infusions of 0.08 to $0.09 \mu \mathrm{g} / \mathrm{kg} / \mathrm{min}$. One patient received dopamine at a maximum dose of $10 \mu \mathrm{g} / \mathrm{kg} / \mathrm{min}$. Five patients $(36 \%)$ underwent treatment with vasopressin, with doses ranging between 0.1 and $0.7 \mathrm{mU} / \mathrm{kg} / \mathrm{min}$. The 3 patients who did not meet criteria within the first 12 hours following transplant all received CF-VAD support pretransplant, and all met criteria for vasoplegia by 24 hours following transplant. Neither of the 2 patients supported with a pulsatile VAD pretransplant developed vasoplegia.

\section{Association With Outcomes}

Overall, $23 \%$ of patients developed stage II or III AKI after heart transplant and $57 \%$ of patients experienced hypotensive events on postoperative day 2 or 3 (Table 3 ). Although more patients with vasoplegia were diagnosed with AKI ( $36 \%$ vs $17 \% ; P=.247)$, the differences were not statistically significant. There were also no significant differences in hospital stay (18 days; IQR, 13-34 days vs
TABLE 2. Frequencies of variables selected at $\boldsymbol{P}=.05$ significance level

\begin{tabular}{lcc}
\hline \multicolumn{1}{c}{ Variable } & $\begin{array}{c}\text { Univariable } \\
\text { model } \\
(\mathbf{n}=\mathbf{1 0 , 0 0 0})\end{array}$ & $\begin{array}{c}\text { Bivariable } \\
\text { model } \\
(\mathbf{n}=\mathbf{8 3 1 1})\end{array}$ \\
\hline Female sex & 9 & 10 \\
\hline Age $(\mathrm{y})$ & 9 & 6 \\
\hline Height $(\mathrm{cm})$ & 16 & 7 \\
Weight $(\mathrm{kg})$ & 8 & 20 \\
\hline Single ventricle & 10 & 54 \\
\hline Congenital heart disease & 10 & 25 \\
\hline Angiotensin converting enzyme inhibitors & 9 & 11 \\
\hline Sprionolactone & 10 & 7 \\
\hline Amiodarone & 16 & 6 \\
Inotropy & 10 & 28 \\
\hline Creatinine (mg/dL) & 9 & 13 \\
\hline Hemoglobin (g/dL) & 10 & 51 \\
\hline Cardiopulmonary bypass time (min) & 7 & 10 \\
\hline Plasmaphresis & 8 & 9 \\
\hline Graft ischemic time (min) & 8 & 13 \\
\hline Continuous flow & 8 & - \\
\hline Values are presented as \%. & &
\end{tabular}

16 days; IQR, $13-20$ days; $P=.472$ ). There was no difference in the median length of ICU stay or duration of mechanical ventilation. Despite differences in pretransplant hemoglobin levels, only 3 out of 14 patients with vasoplegia $(21 \%)$ required blood transfusion during the 72 hours following transplant compared with 9 out of $30(30 \%)$ in the no-vasoplegia group.

\section{DISCUSSION}

This is the first report of the incidence and risk factors for the development of vasoplegia after OHT in children. In this diverse cohort, approximately one-third of patients developed vasoplegia, and the incidence was $>50 \%$ in patients supported preoperatively with a CF-VAD. This compares with $13 \%$ in patients with pretransplant pulsatile blood flow. Aside from CF-VAD use, there were no statistically significant predictors identified for the development of vasoplegia in the univariable analysis (Table 2 and Video 1). In the presence of CF-VAD use, pretransplant single-ventricle physiology also showed an association with vasoplegia greater than other variables evaluated. However, due to the low number of patients with single-ventricle physiology who were supported with a CF-VAD, the study was not powered specifically to evaluate this subset of the cohort. This study was also not powered to comment on the association with adverse outcomes; however, there were trends toward worse outcomes in patients who did develop vasoplegia. 
TABLE 3. Association of vasoplegia with posttransplant outcomes

\begin{tabular}{lcccc}
\hline \multicolumn{1}{c}{ Outcome } & Total population $(\mathbf{N}=\mathbf{4 4})$ & Vasoplegia $(\mathbf{n}=\mathbf{1 4})$ & No vasoplegia $(\mathbf{n}=\mathbf{3 0})$ & $\boldsymbol{P}$ value \\
\hline Acute kidney injury & $10(23)$ & $5(36)$ & $5(17)$ & .247 \\
Duration of mechanical ventilation (d) & $2(1-3)$ & $2(1-4)$ & $2(1-3)$ & .979 \\
Duration of CVICU stay (d) & $8(6-12)$ & $8(6-15)$ & $.362-10)$ & .362 \\
Duration of hospitalization (d) & $16(13-20)$ & $18(13-34)$ & .472 \\
\hline
\end{tabular}

Values are presented as $\mathrm{n}(\%)$ or median (interquartile range). $\mathrm{CVICU}$, Cardiovascular intensive care unit.

This is also the first pediatric study to propose a clinically useful definition for vasoplegia (Figure 2). We believe that the presence of low diastolic pressure with concomitant use of a vasopressor (or inotrope used as a vasopressor) utilizes readily available medical data to allow prospective and retrospective determination of vasoplegia in a variety of clinical scenarios. Although not perfect in retrospective analysis, the requirement of normal systolic heart function, right atrial pressure of at least $5 \mathrm{~mm} \mathrm{Hg}$, and absence of positive blood cultures eliminates many other etiologies for hypotension. This study was not powered to validate this definition and broader investigation is certainly necessary; however, a unified definition of vasoplegia in children is necessary for continued study of this state in pediatric populations.

Knowledge of the association of vasoplegia in patients supported preoperatively with CF-VAD technology is of significant clinical value. Undiagnosed and/or improperly treated vasoplegia can lead to life-threatening hypotensive episodes that may lead to inadequate oxygen delivery and end-organ injury, most commonly in the form of AKI. Moreover, excessive use of fluid resuscitation for these episodes can negatively influence right heart function and may

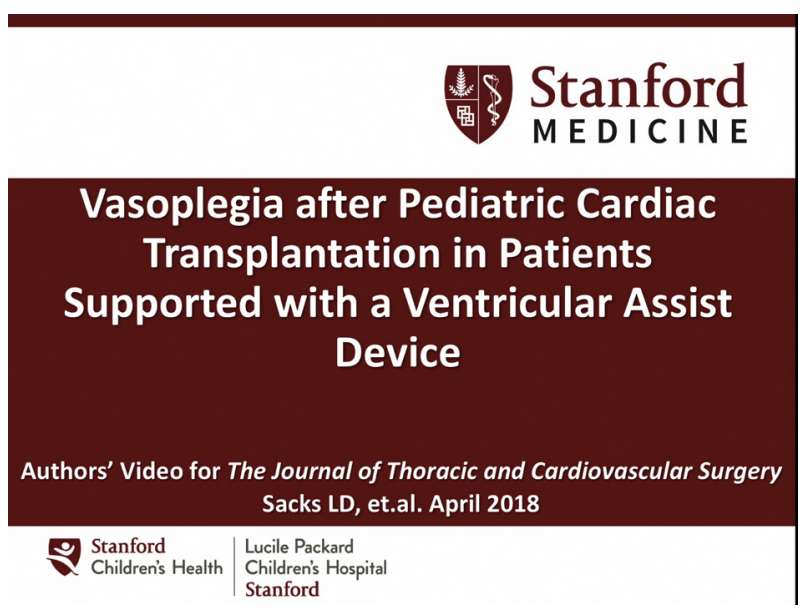

VIDEO 1. Summary video showing how the article provides a significant correlation between the use of continuous flow devices and the incidence of vasoplegia following pediatric heart transplant. In addition, a standardized, clinical definition of vasoplegia is proposed, allowing further research into this phenomenon. Video available at: https://www.jtcvs.org/article/S00225223(19)30315-0/fulltext. prolong posttransplant intubation and ICU stay. Pediatric patients have smaller blood vessels and the use of invasive hemodynamic monitoring devices is rare. Therefore, unlike adult patients, the ability to directly monitor changes in vascular tone is limited. Awareness of this potentially deadly hemodynamic phenomenon allows cardiac intensivists to be prepared for vasoplegic events, bringing appropriate medications to bear on the ever-growing population of children receiving mechanical circulatory support. ${ }^{19}$

Such medications may include methylene blue, a common therapy used in the treatment of catecholamine-refractory vasoplegia following cardiac surgery ${ }^{20,21}$ or after severe burns. ${ }^{22}$ Additionally, mixed success has been found with the use of hydroxocobolamin in the treatment of this pathologic state. ${ }^{23}$ However, the use of vasoconstricting agents such as norepinephrine, vasopressin, and/or high doses of epinephrine or dopamine remain the mainstay of therapy. ${ }^{24}$ In the study center, standard inotropic support following heart transplant consisted of epinephrine 0.02 to $0.04 \mu \mathrm{g} / \mathrm{kg} / \mathrm{min}$, dopamine 3 to $5 \mu \mathrm{g} / \mathrm{kg} / \mathrm{min}$, and milrinone 0 to $0.5 \mu \mathrm{g} / \mathrm{kg} /$ $\mathrm{min}$. The use of increased doses of epinephrine or dopamine, and the use of vasoconstricting agents (ie, vasopressin) is typically pursued only after a clinical diagnosis of vasoplegia is made. These agents may then be utilized until vascular tone is restored and form the primary treatment modality for all patients in this study. No patient received either methylene blue or hydroxocobolamin.

Several adult studies have demonstrated an association of vasoplegia with preoperative CF-VAD support, ${ }^{6-8}$ although these results are not uniform. ${ }^{25,26}$ The largest adult study of 311 patients undergoing transplant demonstrated that preoperative support with a CF-VAD was associated with a $25 \%$ incidence of vasoplegia, 3 times higher than those without CF-VAD support. ${ }^{6}$ Unlike our study, several other variables were found to predict vasoplegia, including donor ischemia time, ${ }^{6,8}$ preoperative inotrope use, ${ }^{6}$ and preoperative creatinine level. ${ }^{27}$ A larger study is necessary to fully evaluate other risk factors in a heterogeneous pediatric population; however, it is striking that this first pediatric study shows only the use of CF-VAD support as a predictor of posttransplant vasoplegia.

Larger adult reports differ from our study in finding the association of vasoplegia with adverse outcomes. The 2 largest adult studies demonstrated that vasoplegia was associated with longer ICU stay and duration of mechanical 
ventilation, higher mortality, and higher incidence for reoperation and extracorporeal membrane oxygenation. ${ }^{6,26}$ Another study looking at long-term outcomes found that patients with vasoplegia did not have a difference in 1-year survival or rates of rejection despite findings of worse short-term morbidity. ${ }^{8}$ Similarly, vasoplegia is shown to be associated with increased morbidity and mortality in other populations of adults and children undergoing cardiac surgery. ${ }^{9,28,29}$ It is possible that, with a larger cohort, association with adverse outcomes may have been seen.

The mechanism of postoperative vasoplegia following orthotopic heart transplant is multifactorial. Inflammation due to cardiopulmonary bypass, blood product administration, and surgical manipulation may lead to vasodilation after routine cardiac surgery. ${ }^{26}$ It has also been demonstrated that inflammation plays an integral role in endothelial dysfunction in patients with heart failure, ${ }^{30}$ and that chronic heart failure leads to neurohormone activation and an increased sympathetic tone that may blunt feedback mechanisms. ${ }^{31}$ Additionally, it has been demonstrated that prolonged nonpulsatile blood flow is an independent risk factor for vasoplegia. Comparison of patients with pulsatile and continuous flow VADs demonstrated that those with CF-VADs had elevated serum proinflammatory cytokines, ${ }^{32}$ and pronounced vascular changes with inflammatory changes including T-cell and macrophage accumulations and increased expression of proinflammatory vascular cell adhesion molecules. ${ }^{33}$ Furthermore, it has been postulated that continuous flow causes autonomic dysfunction due to chronic baroreceptor unloading. ${ }^{34}$ The combination of chronic heart failure, nonpulsatile blood flow, and extensive surgical dissection necessary for pediatric heart transplantation may all contribute to the predisposition to vasoplegia in this cohort.

This study has the benefit of including a relatively large pediatric cohort at a center with extensive experience in OHT and VADs. However, several limitations apply: The cohort was not large enough to identify other risk factors for vasoplegia or to find associations with outcomes. Moreover, the study was limited by the low number of patients in the study cohort supported with pulsatile devices before transplant. Unlike adult populations, invasive measurements of cardiac index and vascular resistance are not readily available for postoperative monitoring and management. Therefore, the clinical diagnosis of vasoplegia relied primarily on the monitoring of blood pressure and physical exam. In this retrospective chart review, blood pressure reporting was limited to discreet times, and was influenced by multiple factors, including cardiac systolic dysfunction, bleeding, and other volume depletion. In this study medication selection was used as a surrogate for physician judgment. It was assumed that vasopressin, norepinephrine, and high-dose epinephrine or dopamine were used in the setting of exam findings consistent with low vascular tone. Unfortunately, these medications may also be used in other states of hypotension. Echocardiographic evaluation of heart function was performed daily following transplant according to the standard management protocol of the study institution. Given that this was not tied to specific changes in clinical condition, it may be that vasoconstriciting medications/doses were initiated at the time heart function was transiently depressed. However, even if this were the case, these results would indicate a propensity to hypotension in the CF-VAD population that is refractory to the standard inotropic medications used in the study center. There is also the potential that providers are more likely to be vigilant for vasoplegia in patients with CF-VADs biasing toward use in this cohort. Enrollment was limited to age older than 3 years to focus our evaluation on a population for which CF-VADs were regularly utilized; therefore, further evaluation in younger ages is necessary to evaluate risk factors and outcomes associated with vasoplegia after OHT in infants.

\section{CONCLUSIONS}

The use of a CF-VAD is associated with the development of vasoplegia following OHT in children. Within our limited sample, pediatric patients who developed postoperative vasoplegia were not more likely to develop AKI, or have prolonged duration of mechanical ventilation or cardiac ICU stay. Awareness of the association between CF-VAD use and posttransplant vasoplegia may allow providers to improve their vigilance for a phenomenon that has been previously shown to have potentially deadly consequences. Larger, multicentered studies are necessary to further understand risk factors, outcomes, and treatment for vasoplegia in this rapidly evolving patient population.

\section{Conflict of Interest Statement}

Authors have nothing to disclose with regard to commercial support.

\section{References}

1. Dipchand AI, Kirk R, Edwards LB, Kucheryavaya AY, Christie JD, Dipchand AI, et al. The Registry of the International Society for Heart and Lung Transplantation: sixteenth official pediatric heart transplantation report-2013; focus theme: age. J Heart Lung Transplant. 2013;32:979-88.

2. Adachi I, Burki S, Horne D, Jeewa A, Elias B, McKenzie E, et al. Continuous flow VAD support at a tertiary pediatric center: compared to PediMACS data. J Heart Lung Transplant. 2017;36:S280-1.

3. Rossano JW, Lorts A, VanderPluym CJ, Jeewa A, Guleserian KJ, Kleiweis MS, et al. Outcomes of pediatric patients supported with continuous-flow ventricular assist devices: a report from the Pediatric Interagency Registry for Mechanical Circulatory Support (PediMACS). J Heart Lung Transplant. 2016;35:585-90.

4. Kirklin JK, Naftel DC, Pagani FD, Kormos RL, Stevenson LW, Blume ED, et al. Sixth INTERMACS annual report: a 10,000-patient database. J Heart Lung Transplant. 2014;33:555-64.

5. Miller JR, Lancaster TS, Epstein DJ, DuPont NC, Simpson KE, Castleberry C, et al. Outcomes and trends of ventricular assist device selection in children with end-stage heart failure. ASAIO J. 2017;63:464-9.

6. Patarroyo M, Simbaqueba C, Shrestha K, Starling RC, Smedira N, Tang WH, et al. Pre-operative risk factors and clinical outcomes associated with vasoplegia 
in recipients of orthotopic heart transplantation in the contemporary era. J Heart Lung Transplant. 2012;31:282-7.

7. Amir O, Radovancevic B, Delgado RM III, Kar B, Radovancevic R, Henderson M, et al. Peripheral vascular reactivity in patients with pulsatile vs axial flow left ventricular assist device support. J Heart Lung Transplant. 2006;25:391-4.

8. Chan JL, Kobashigawa JA, Aintablian TL, Li Y, Perry PA, Patel JK, et al. Vasoplegia after heart transplantation: outcomes at 1 year. Interact Cardiovasc Thorac Surg. 2017;25:212-7.

9. van Vessem ME, Palmen M, Couperus LE, Mertens B, Berendsen RR, Tops LF, et al. Incidence and predictors of vasoplegia after heart failure surgery. Eur J Cardiothorac Surg. 2017;51:532-8.

10. Omar S, Zedan A, Nugent K. Cardiac vasoplegia syndrome: pathophysiology, risk factors and treatment. Am J Med Sci. 2015;349:80-8.

11. Lemson J, Nusmeier A, van der Hoeven JG, Lehman R, Ceccheti C. The pulmonary artery catheter in the pediatric intensive care unit: not the way to go. Pediatr Crit Care Med. 2012;13:250-1.

12. Wong HR, Dalton HJ. The PICU perspective on monitoring hemodynamics and oxygen transport. Pediatr Crit Care Med. 2011;12:S66.

13. Harris PA, Taylor R, Thielke R, Payne J, Gonzalez N, Conde JG. Research electronic data capture (REDCap) — a metadata-driven methodology and workflow process for providing translational research informatics support. J Biomed Inform. 2009;42:377-81.

14. Haque IU, Zaritsky AL. Analysis of the evidence for the lower limit of systolic and mean arterial pressure in children. Pediatr Crit Care Med. 2007;8: $138-44$.

15. National High Blood Pressure Education Program Working Group on High Blood Pressure in Children and Adolescents. The fourth report on the diagnosis, evaluation, and treatment of high blood pressure in children and adolescents. $\mathrm{Pe}$ diatrics. 2004;114:555-76.

16. Kellum JA, Lameire N. Diagnosis, evaluation, and management of acute kidney injury: a KDIGO summary (Part 1). Crit Care. 2013;17:204

17. Gaies MG, Jeffries HE, Niebler RA, Pasquali SK, Donohue JE, Yu S, et al. Vasoactive-inotropic score is associated with outcome after infant cardiac surgery: an analysis from the Pediatric Cardiac Critical Care Consortium and virtual PICU system registries. Pediatr Crit Care Med. 2014;15:529-37.

18. Gaies MG, Gurney JG, Yen AH, Napoli ML, Gajarski RJ, Ohye RG, et al. Vasoactive-inotropic score as a predictor of morbidity and mortality in infants after cardiopulmonary bypass. Pediatr Crit Care Med. 2010;11:234-8.

19. Davies RR, Russo MJ, Hong KN, O’Byrne ML, Cork DP, Moskowitz AJ, et al. The use of mechanical circulatory support as a bridge to transplantation in pediatric patients: an analysis of the United Network for Organ Sharing database. $J$ Thorac Cardiovasc Surg. 2008;135:421-7.e421.

20. Del Duca D, Sheth SS, Clarke AE, Lachapelle KJ, Ergina PL. Use of methylene blue for catecholamine-refractory vasoplegia from protamine and aprotinin. Ann Thorac Surg. 2009;87:640-2.
21. Evora PR, Levin RL. Methylene blue as drug of choice for catecholaminerefractory vasoplegia after cardiopulmonary bypass. J Thorac Cardiovasc Surg. 2004; 127:895-6.

22. Church JT, Posluszny JA, Hemmila M, To KB, Cherry-Bukowiec JR, Waljee J. Methylene blue for burn-induced vasoplegia: case report and review of literature. J Burn Care Res. 2015;36:e107-11.

23. Shah PR, Reynolds PS, Pal N, Tang D, McCarthy H, Spiess BD. Hydroxocobalamin for the treatment of cardiac surgery-associated vasoplegia: a case series. Can J Anaesth. 2018;65:560-8.

24. Levy B, Fritz C, Tahon E, Jacquot A, Auchet T, Kimmoun A. Vasoplegia treatments: the past, the present, and the future. Crit Care. 2018;22:52.

25. Chemmalakuzhy J, Costanzo M, Meyer P, Piccione W, Kao W, Winkel E, et al. Hypotension, acidosis, and vasodilatation syndrome post-heart transplant: prognostic variables and outcomes. J Heart Lung Transplant. 2001;20:1075-83.

26. Byrne JG, Leacche M, Paul S, Mihaljevic T, Rawn JD, Shernan SK, et al. Risk factors and outcomes for 'vasoplegia syndrome'following cardiac transplantation. Eur J Cardiothorac Surg. 2004;25:327-32.

27. Kao C-H, Lin C-Y, Lu C-W, Tsai Y-T, Lee C-Y, Lin Y-C, et al. Vasoplegia syndrome is not associated with higher mortality rate after heart transplant. Acta Cardiol Sin. 2012;28:129-36.

28. Levin MA, Lin H-M, Castillo JG, Adams DH, Reich DL, Fischer GW. Early oncardiopulmonary bypass hypotension and other factors associated with vasoplegic syndrome. Circulation. 2009;120:1664-71.

29. Rosenzweig EB, Starc TJ, Chen JM, Cullinane S, Timchak DM, Gersony WM, et al. Intravenous arginine-vasopressin in children with vasodilatory shock after cardiac surgery. Circulation. 1999;100(19 Suppl):II182-6.

30. Tousoulis D, Charakida M, Stefanadis C. Inflammation and endothelial dysfunction as therapeutic targets in patients with heart failure. Int J Cardiol. 2005;100: 347-53.

31. Cohn JN, Levine TB, Olivari MT, Garberg V, Lura D, Francis GS, et al. Plasma norepinephrine as a guide to prognosis in patients with chronic congestive heart failure. N Engl J Med. 1984;311:819-23.

32. Loebe M, Koster A, Sänger S, Potapov EV, Kuppe H, Noon GP, et al. Inflammatory response after implantation of a left ventricular assist device: comparison between the axial flow MicroMed DeBakey VAD and the pulsatile Novacor device. ASAIO J. 2001;47:272-4.

33. Lee M, Akashi H, Kato TS, Takayama H, Wu C, Xu K, et al. Vascular inflammation and abnormal aortic histomorphometry in patients after pulsatile-and continuous-flow left ventricular assist device placement. J Heart Lung Transplant. 2016;35:1085-91.

34. Markham DW, Fu Q, Palmer MD, Drazner MH, Meyer DM, Bethea BT, et al. Sympathetic neural and hemodynamic responses to upright tilt in patients with pulsatile and nonpulsatile left ventricular assist devices. Circ Heart Fail. 2013; 6:293-9.

Key Words: vasoplegia, VAD, heart transplant, pediatrics 